\title{
An unusual cause of dementia
}

\author{
Michael Vassallo, Steve Allen
}

\begin{abstract}
Summary
Gliomatosis cerebri is a rare cerebral tumour that presents with personality and mental state changes. Diagnosis can be very difficult and many times is made at post mortem. We describe a 63-yearold man who presented initially with depression that merged into a schizophrenia-like illness, and who developed progressive dementia prior to his death. Two computed tomography (CT) scans of the brain were normal and the diagnosis of gliomatosis cerebri was made at post mortem. The progressively changing mental state was suggestive of an organic cause of his illness. Since this tumour may not be detected by a CT scan, a magnetic resonance imaging scan with T2-weighted images with spin echo sequences of the brain should be performed. Prognosis is very poor but diagnosis is important to plan terminal care. The patient described was unusual because he was older than most people with this tumour, and he presented with psychiatric symptoms which were thought to be non-organic for almost two years.
\end{abstract}

Keywords: dementia, gliomatosis cerebri

Astrocytic tumours are common, accounting for nearly $50 \%$ of all brain tumours. Classification is controversial. Classically, they have been classified in the Kernohan classification into four grades depending on their degree of malignancy. However, more recently several three-tier grading schemes based on histologic criteria have been suggested. ${ }^{1}$ Gliomatosis cerebri is of uncertain astrocytic origin. Clinical progression varies from a few days to several years. Personality and mental state changes are the most common presenting features and the clinical signs reflect the diffuse pathological nature of the tumour. Computed tomographic (CT) scans of the brain can be negative and diagnosis is difficult. The present case highlights some of these difficulties.

Medicine for of

Elderly, Royal

Bournemouth

Hospital, Castle Lane

East, Bournemouth, Dorset BH7 7DW, UK $M$ Vassallo

S Allen

Correspondence to Dr S Allen

Accepted 15 February 1995 antidepressant treatment, he developed a schizophrenic clinical picture with severe delusions of grandeur, paranoia, auditory hal- lucinations and thought blocking. There was a good initial response to treatment with haloperidol by a psychiatrist. Gradually, he lost his intellectual function. Over the subsequent six months he lost social graces and became progressively demented. There was a strong family history of presenile dementia, affecting his sister and mother, both of whom were deceased though no post mortem information was available. His paranoia relapsed and he made no further improvement with anti-psychotic medication. At this stage he was referred to the authors by his psychiatrist because organic brain disease was suspected. Physical examination at this stage revealed a cachectic Caucasian with onychogryphosis of the toe nails. There was evidence of severe cognitive impairment and his plantar responses were extensor, but the rest of the physical examination was unremarkable. His full blood count, liver, renal and thyroid function tests were normal, as were treponemal serology and serum vitamin B 12 and folate. Two CT scans of the brain with contrast in 1991 (when the psychosis began) and 1993 (when the dementia began) were reported as showing minimal thalamic calcification but no evidence of cerebral infarction, atrophy or space-occupying lesions; they were deemed to be within normal limits by two radiologists. Clinically, pre-senile dementia was suspected, particularly Pick's disease, because of the frontal and temporal lobe features though the absence of atrophy on the CT scan was not in keeping with this diagnosis. $\mathrm{He}$ continued to deteriorate and eventually succumbed to a bronchopneumonia, aged 66 .

A post mortem was performed since the diagnosis was uncertain and to facilitate the possible need for genetic counselling. The brain was of normal weight and external examination was unremarkable. Histology showed gliomatosis cerebri, a diffusely invading astrocytoma infiltrating many areas of the frontal lobe. A large pontine haemorrhage was thought to be secondary to brainstem distortion by the tumour and was the actual cause of death.

\section{Discussion}

Gliomatosis cerebri is of uncertain astrocytic origin, consisting of a diffuse astrocytic proliferation of varying grade involving the cerebral hemispheres, brainstem and cerebellum. The neoplastic cells infiltrate extensively, but neuronal elements are preserved except in areas where the infiltration becomes very dense. Different theories of origin and spread have 
been postulated. The most widely accepted theory suggests that the tumour arises from multicentric transformation (dedifferentiation) of preexisting astrocytes with subsequent centrifugal spread. ${ }^{2}$ There are two peaks of frequency, between $10-20$ and $40-50$ years. Our patient was unusually old to present with this particular form of astrocytoma. Clinical signs reflect the diffuse pathological nature of the tumoural process. The range of clinical progression has been reported to last from 25 days to 20 years. $^{3}$ Personality and mental state changes with dementia are the most frequently reported clinical symptoms although other symptoms are also encountered (see box). ${ }^{4}$ Our patient was unusual in presenting with depression evolving into a clinical illness indistinguishable from paranoid schizophrenia, until he started to develop dementia and frontotemporal features.

Diagnosis is difficult. Plain skull radiography, radionucleotide brain scans, angiography, pneumoencephalography and ventriculography are not helpful. An electroencephalogram most commonly shows diffuse slow activation with varying amounts of focal slowing or occasional spikes. Cerebrospinal fluid sometimes shows small elevations of protein but only rarely are there any cells. CT scans may be negative or they may show diffuse white matter hypodensity, with small ventricles, little mass effect, diffuse enlargement of the cerebral parenchyma and shallow cortical sulci. Enhancement is usually minimal or absent, suggesting that the blood-brain barrier is usually preserved. ${ }^{5}$ Plausible explanations for an early negative appearance on CT include small tumour size, low cell density, isodense tumour mass, lack of acquired changes in the blood brain barrier or its stabilization by pre-scan steroid medication and poor scan resolution. The influence of these factors increases when dealing with low-grade diffusely infiltrating lesions ${ }^{6}$ such as the one described in this case. Magnetic resonance imaging (MRI) displays a diffuse high-density signal or poor grey/white matter demarcation on T2-weighted spin-echo images. MRI seems to be indispensible for the study of astrocytic tumours and it is the only available method of confirming low-grade lesions that have passed unnoticed or have merely been suspected at CT scan. ${ }^{3} \mathrm{MRI}-$ autopsy correlation suggests that the extent of infiltration may be imperfectly assessed by current imaging modalities. ${ }^{5}$ The main differ-

1 Daumas-Duport C, Scheithauer B, O'Fallon J, Kelly P. Grading of astrocytomas: a simple and reproducible method. Cancer 1988; 62: 2152-65.

2 Geremia GK, Wollman R, Foust R. Computed tomography of gliomatosis cerebri: case report. f Comput Assist Tomogr 1988; 12: 698-701.

3 Margain D, Peretti-Viton P, Arnaud O, Martini P, Salamon G. Astrocytic tumours. $\mathcal{f}$ Neuroradiol 1991; 18: 141-52.

4 Couch JR, Weiss SA. Gliomatosis cerebri: report of four cases and review of the literature. Neurology 1974; 24: cases and

\section{Clinical features of gliomatosis} cerebri

- variable clinical progression from 25 days to 20 years

- commonly presents with personality or mental state changes including a changing psychiatric clinical picture with dementia

- other clinical feature include hemiparesis, ataxia, headache, raised intracranial pressure, cranial nerve palsies, epileptic seizures, visual loss, nausea and vomiting

- CT scans may be negative and MRI may be the only available non-invasive method of confirming low-grade lesions

- prognosis is poor and the diagnosis is usually made at post mortem

\section{Learning/summary points}

- differential diagnosis includes leucodystrophy, lymphoma, metastases, and multicentric glioblastoma

- diagnosis sometimes requires a very high index of clinical suspicion

- a rapidly changing psychiatric clinical picture can be the only clue leading to the diagnosis

- despite the dismal prognosis, diagnosis is worth persuing to plan terminal care

ential diagnosis is leucodystrophy, lymphoma, metastases, and multicentric glioblastoma.

Our case highlights the difficulties of diagnosing this condition, with two normal CT scans done at an interval of two years. At post mortem the diagnosis was confirmed only after histological examination. In retrospect, the rapdily changing psychiatric picture was the most important clue to the underlying organic nature of this patient's condition. Ante mortem diagnosis is important since it helps plan management, even though prognosis is hopeless. To avoid missing these tumours adults presenting with an unusual psychiatric picture, especially if they fail to respond to conventiona treatment, should have an MRI using T2 weighted spin-echo sequences.

We acknowledge the assistance of Dr M Rowton-Lee of St Ann's Hospital, Poole, Dorset and Dr DW Ellison from the Wessex Neurological Centre, Southampton.

5 Koslow SA, Claassen D, Hirsch WL, Jungreis CA Gliomatosis cerebri: a case report with autopsy correlation. Neuroradiology 1992; 34: 331-3.

6 Hylton PD, Reichman OH. Clinical manifestation of gliom before computed tomographic appearance: the dilemma of a negative scan. Neurosurgery 1987; 21: 27-31. 